\title{
Symmetry-protected topological phases and orbifolds: Generalized Laughlin's argument
}

\author{
Olabode Mayodele Sule, ${ }^{1}$ Xiao Chen, ${ }^{1}$ and Shinsei Ryu ${ }^{1}$ \\ ${ }^{1}$ Department of Physics, University of Illinois at Urbana-Champaign, \\ 1110 West Green St, Urbana, Illinois 61801, USA
}

( $\Omega$ Dated: September 19, 2018)

\begin{abstract}
We consider non-chiral symmetry-protected topological phases of matter in two spatial dimensions protected by a discrete symmetry such as $\mathbb{Z}_{K}$ or $\mathbb{Z}_{K} \times \mathbb{Z}_{K}$ symmetry. We argue that modular invariance/noninvariance of the partition function of the one-dimensional edge theory can be used to diagnose whether, by adding a suitable potential, the edge theory can be gapped or not without breaking the symmetry. By taking bosonic phases described by Chern-Simons K-matrix theories and fermionic phases relevant to topological superconductors as an example, we demonstrate explicitly that when the modular invariance is achieved, we can construct an interaction potential that is consistent with the symmetry and can completely gap out the edge.
\end{abstract}

\section{INTRODUCTION}

Topological phases are highly quantum states of matter that have no classical analog. The earliest realization of a genuine topological phase is the quantum Hall effect (QHE). Since the discovery of the QHE, many exotic properties of topological phases, their realizations beyond the QHE in two-dimensional electron gas (e.g., in the context of spin liquids), and their potential use in quantum computation, etc., have actively been studied both theoretically and experimentally. $\underline{1-3}$

Although symmetry does not play much role in the QHE, an interplay between topology and symmetry can, and does, play an important role in the physics of topological phases. A symmetry-protected topological (SPT) phase is a short-ranged entangled state of matter with trivial topological order but with symmetry. That is, in the absence of symmetry all such phases can be connected adiabatically to one another. With symmetry, however, they are distinct phases that cannot be adiabatically connected to one another without breaking the symmetry. Examples of SPTs include, for example, the Haldane phase in one-dimensional quantum spin chains, the quantum spin Hall effect, and the threedimensional time-reversal-symmetric topological insulator and superconductors. $\stackrel{4-16}{-16}$

In one spatial dimension $(\mathrm{d}=1)$, (topological) properties of gapped phases can be conveniently studied in terms of the tensor-network (matrix-product state) representation of the ground-state wave functions ${ }^{17-24}$ By utilizing such representation of the ground-state wave functions, it has been shown that all SPT phases in $d=1$ with a given symmetry group $\mathrm{G}$ can be fully classified ${ }^{17-20}$ In higher dimensions $d>1$, a large class of ground-state wave functions of SPT phases can be constructed using the tensor-network method $\stackrel{25,26}{2}$ For SPT phases that can be realized by combining Abelian topological phases in two dimensions, Chern-Simons K-matrix theories have been adopted to construct, characterize, and classify various SPT phases ${ }^{27-32}$ Another theme involves using the braiding statistics of quasiparticle excitations in the bulk to classify the SPT phases. ${ }^{33-37}$

The purpose of this paper is to discuss SPT phases in $2+1$ dimensions $[(2+1) \mathrm{D}]$ that are accompanied by stable edge states, following the approach proposed in Ref. 38. Suppose we have a $(1+1)$ dimensional conformal field theory (CFT), that may be realized as an edge state of a (topological) bulk theory. The CFT can be either chiral or non-chiral, but our main focus will be on non-chiral edge theories. Let us also assume the CFT is invariant under some global on-site symmetry group $G$. We then ask if the gapless nature of the CFT is stable or not against perturbations once the symmetry $G$ is enforced. It the CFT cannot be gapped without breaking the symmetry $G$, it cannot exist on its own as an isolated $(1+1)$-dimensional system, but it must be realized as an edge theory of a bulk SPT protected by symmetry group $G$.

The strategy suggested in Ref. 38 consists of the following two ingredients. The first ingredient is the strict enforcement of the symmetry by a projection operation on the CFT. (A similar projection or gauging procedure is also employed in Ref 36 in a related context - see below.) The second ingredient is the modular (non) invariance of the projected edge CFTs. This can be thought of as a geometrical (or: gravitational) generalization of Laughlin's thought experiment $\stackrel{39}{ }$ In the Laughlin argument, an insertion of an integer multiple of flux quanta in a quantum Hall system on the cylinder geometry (say the ends of a cylinder) results in a transport of charge excitations from one edge to another, i.e., Thouless pumping. This leads to a non-conservation (quantum anomaly) of particle number within the single edge theory and can thus be viewed as a signature of the presence of a non-trivial bulk. This idea can be generalized to include modular transformations which are large coordinate transformations in CFTs.

Within our approach, the characterization and classification of SPT phases can be viewed as those of (nonchiral) CFTs with symmetry projection. Such CFTs are obtained from a projection by a (discrete) group and are usually called orbifold CFTs ${ }^{40-42}$ It is often the case that 
the symmetry-projected theory can be made modular invariant as far as the symmetry group acts on holomorphic and anti-holomorphic sectors of the CFT in a symmetric fashion. Once the symmetry group acts on the holomorphic and anti-holomorphic sectors in an assymetric way (e.g., as an extreme example, $G$ can act solely on the holomorphic sector but not on the anti-holomorphic sector) the modular invariance is no longer guaranteed. Such orbifold is called an asymmetric orbifold $\underline{\underline{43}}$ Our approach may then be summarized as " $(2+1) \mathrm{D}$ SPT phases $=$ asymmetric orbifolds", as a slogan $\underline{\underline{42}}$

The structure of the paper and the main results are summarized as follows. In Sec. II we start our discussion by giving a more general discussion of modular invariance and gauging symmetries in SPT phases.

In Sec.III, we discuss bosonic SPT phases described by Chern-Simons K-matrix theories in the bulk with $\mathbb{Z}_{K} \times \mathbb{Z}_{K}$ symmetry. With $N_{f}$ copies (flavors) of the edge theories, we find that modular invariance can be achieved when there are $N_{f}=0 \bmod K$ flavors. This suggests the phases are trivial if and only if (iff) $N_{f}=0$ $\bmod K$. When $N_{f}=1$ and for a particular $\mathbb{Z}_{K}$ subgroup of $\mathbb{Z}_{K} \times \mathbb{Z}_{K}$ we also reproduce within the modular (non)invariance approach some of the results based on a K-matrix formulation used in Ref. 29.

Similarly, in Sec. IV, we study fermionic phases with $\mathbb{Z}_{K} \times \mathbb{Z}_{K}$ symmetry. We find that modular invariance can be achieved when $N_{f}=2 K(\bmod 2 K)$. Here $N_{f}$ refers to the number of flavors of complex Dirac fermions. This generalizes the earlier results in Refs. 38 and 44 . (See Ref. 45 for a similar model.) In all cases considered we analyze the stability of the edge states to gapping when interactions are considered and explicitly construct $\left(\mathbb{Z}_{K} \times \mathbb{Z}_{K}\right)$-invariant potentials that can completely gap out the edge states whenever the phase is trivial. Finally, we conclude in Sec. V with a summary of our results and an outlook for future applications.

\section{MODULAR INVARIANCE AND GAUGING SYMMETRY IN SPT PHASES}

a. Laughlin's thought experiment We start by giving a brief overview of our approach to $(2+1)$ dimensional SPT phases, which may be viewed as an gravitational analog of Laughlin's thought experiment $\underline{\underline{39}}$

Let us first recall some key steps in Laughlin's thought experiment. Consider a quantum Hall system on a finite cylinder with two edges, I and II. If magnetic flux $\Phi$ is threaded adiabatically through the cylindrical hole, as the flux is increased (adiabatically), starting from, say, zero flux, the Hamiltonian $H(\Phi)$ of the system is in general not invariant under the flux insertion. However, after an increase by an integral multiple of the flux quantum $\Phi_{0}$, the Hamiltonian comes back to itself. That is, $H\left(\Phi+n \Phi_{0}\right)=H(\Phi)$, for any integer $n$. This is a symmetry of the system but cannot be achieved by successive applications of infinitesimal gauge transformations.
In the process of increasing the flux, an integer multiple of electric charges actually get pumped from one edge to the other due to the QHE. One can analyze this from the point of view of the partition function for the excitation spectrum of the system. Since the transformation is adiabatic and the bulk spectrum is gapped it is enough to focus on the partition function of the edge excitations which have a gapless spectrum. This is given by

$$
Z(\Phi)=\sum_{a, b} N_{a b} \chi_{a}^{\mathrm{I}}(\Phi) \chi_{b}^{\mathrm{II}}(\Phi)
$$

where $\chi_{a}^{\mathrm{I}, \mathrm{II}}$ is a chiral contribution to the partition function for each edge and $N_{a b}$ are constants. Under a large gauge transformation in general, the chiral parts of the partition function is not invariant, $\chi_{a}\left(\Phi+n \Phi_{0}\right) \neq \chi_{a}(\Phi)$, while the total partition function is invariant. This is to be expected for a system with non-trivial bulk and signals the non-conservation of charge $\underline{\underline{46}}$ In the case of the quantum spin Hall effect, a similar flux threading argument ${ }^{27.47}$ can be applied to show that a flux change by $\Phi_{0} / 2$ pumps fermion number parity and leads to spin charge separation.

In the cases we will be interested in, since the total charge may not necessarily be conserved, we look for an alternative to the flux $\Phi$. As advocated in the Introduction, a natural thing to do is to simply replace $\Phi$ by the modular parameter of the torus $\tau$.

b. CFT and modular invariance The effective continuum theory describing the gapless edge state of a bulk (topological) phase is a CFT. A CFT is a field theory defined on a Riemann surface, i.e., a surface with a choice of complex structure (complex coordinates). Two complex structures on a surface are equivalent if there is a biholomorphic map between them. Classically a CFT is invariant under a transformation between equivalent complex structures.

In physical terms CFTs are usually called scale invariant theories, the scale invariance here can be thought of as a rescaling of the metric. On a 2-dimensional surface there is a one to one correspondence beween the space of conformal classes of metrics (with metrics related by a rescaling considered equivalent) and the space of complex structures. Thus a rescaling of the metric can be thought of as a small gauge transformation which via the correspondence is simply a holomorphic(complex-analytic) change of coordinates. After quantization the rescaling invariance of CFTs suffer an anomaly, the trace anomaly proportional to the central charge.

A more general type of gauge transformation is the so called large gauge transformation that cannot be continuously deformed to the identity. On the simplest lower genus surfaces like the two-sphere and the plane there are no large conformal gauge transformations. If one considers the torus one finds that the set of all complex tori (tori with complex structure) are in correspondence with points in the upper half plane. A generic point $\tau$ in the upper half plane represents a torus. It turns out that 
under a transformation

$$
\tau \rightarrow \frac{a \tau+b}{c \tau+d}
$$

with $a, b, c, d \in \mathbb{Z}$ and $a d-b c=1$ one obtains a torus that is equivalent in the sense defined above. These are the large gauge (holomorphic coordinate) transformations on a torus and are referred to as modular transformations.

Modular invariance is the statement of invariance under the transformation (2) and it is usually taken as a constraint on any CFT, if it is derived as the continuum limit of a two-dimensional lattice statistical mechanical system, or a (1+1)-dimensional lattce quantum system. $\underline{\underline{49}}$ Modular invariance can be thought of as a basic constraint that ensures that a CFT is invariant under large gauge transformations at higher genus. Amongst other things it imposes some constraints on the operator content of any CFT, This is the case because a CFT on higher genus can always be constructed from CFT on lower genus by sewing and cutting surfaces, $\underline{50}$ and the large gauge transformations at higher genus are related to those at lower genus. Modular transformations are generated by the so called Dehn twists $\tau \rightarrow \tau+1$ and $\tau \rightarrow-1 / \tau$. In terms of the usual representation of a torus as a doughnut the second transformation exchanges the two nontrivial cycles and can be thought of as an exchange of space and time.

For our application to the edge states, we take our $(2+1)$ - dimensional bulk systems to exist on a cylinder as before. This means that each edge is topologically $S^{1}$ and as far as thermal physics is concerned, the Euclidean field theory for the gapless edge states is a CFT on a geometry which is topologically a torus $S^{1} \times S^{1}$. There is an obvious analogy between the large gauge transformations in the original Laughlins argument and the large coordinate transformation, i.e., the modular transformations. We are thus led to claim that inability to achieve modular invariance is a signal of a nontrivial bulk: Focusing on a single-edge theory (edge I or II in the above discussion), if one cannot construct a modular-invariant partition function within the edge theory, the edge theory cannot be gapped. In other words, such a theory cannot be realized as an isolated $(1+1)$-dimensional system and must be realized as an edge theory of a nontrivial $(2+1)$ dimensional bulk state. Conversely, if one can achieve modular invariance, the edge theory can be gapped.

c. symmetry projection The argument given above so far has not referred to the role played by symmetries. To discuss SPT phases, we now discuss the interplay between symmetry conditions in SPT phases and the modular (non)invariance.

Quite often, a non-chiral CFT can be made modular invariant, once holomorphic and antiholomorphic sectors are properly combined. The non-chiral edge theory of a SPT phase is no exception if symmetry conditions are not enforced - without enforcing symmetries, a SPT phase is adiabatically deformable to a trivial state. There may however be a conflict between modular invariance and symmetry conditions, as the latter may forbid particular ways of combining the left- and right-moving parts of the CFT.

Our strategy to diagnose and characterize a SPT phase is then, to impose the symmetry conditions strictly and ask if the system is invariant under modular transformations or not. More specifically, we take only a single-edge theory (edge I or II in the above notation) and ask if it can be made modular invariant. (If we consider the two separate edges of the modular anomalous theories, they can be combined in a modular-invariant way.) We achieve the strict enforcement of the symmetry by considering a projection of the CFT by the symmetry group G. We now take a close look at the projection procedure.

Let us consider a $1+1 \mathrm{D}$ edge theory with field $\phi$ that has some global symmetry. We work on the torus and given a finite subgroup $G$ of the center of the global symmetry group, we would like to "gauge" $G$. In other words, we would like to consider a field theory with the same Hamiltonian as before but with Hilbert space restricted to the $G$ invariant subspace of the original (this is valid because the Hamiltonian takes $G$ invariant states to $G$ invariant states). In the Hilbert space formalism, the restriction to $G$ invariant states is the same thing as a projection, i.e the partition function in the $G$ invariant sector is

$$
Z=\operatorname{Tr} P e^{-t H},
$$

where $P=|G|^{-1} \sum_{g \in G} \hat{g}$ is a projection operator satisfying $P^{2}=P$, as is easily verified. In the path integral formalism this has the interpretation as a sum over all fields twisted in the time direction of the torus i.e

$$
Z=\sum_{g \in G} \int \mathcal{D}\left[\phi^{g}\right] e^{-S[\phi]},
$$

where the subscript $g$ indicates that the path integral is over fields satisfying

$$
\phi\left(x+2 \pi \tau_{1}, t+2 \pi \tau_{2}\right)=g \phi(x, t) g^{-1} .
$$

This is the meaning of twisting in the time direction. (Here in addition to the translation in the time direction by $2 \pi \tau_{2}$, a finite shift $2 \pi \tau_{1}$ in the spatial direction is also added. This is necessary to discuss a CFT defined on a general torus specified by the modular parameter $\tau$. These two parameters are combined into the modular parameter $\tau, \tau=\tau_{1}+i \tau_{2}$.)

Since the modular invariance is a constraint for a gapless edge theory one needs to ensure that that the partition function (4) is modular invariant. Considering that $\mathrm{S}$ modular transformations interchange space and time it is clear that to achieve modular invariance one would have to explicitly include sectors in the path integral twisted in the spatial direction. [On the other hand, the T modular invariance essentially imposes a constraint on the spectrum of dimensions (hence on operators) in the "gauged" 
(projected) theory.] One can propose that a suitable partition function for the gauged theory is given by

$$
Z=\sum_{(h, g) \in G^{2}} \int \mathcal{D}\left[\phi_{g}^{h}\right] e^{-S[\phi]}
$$

where now the subscript $(h, g)$ indicates that the path integral is restricted to fields twisted in the spatial and time directions by $h$ and $g$ respectively: In addition to the boundary condition (5), the field also obeys

$$
\phi(x+2 \pi, t)=h \phi(x, t) h^{-1}
$$

In general, if the space of all possible field configurations in the path-integral formulation of a quantum field theory is disconnected, the partition function is given as a sum over all disconnected sectors, $Z=\sum_{n} \int \mathcal{D}\left[\phi_{n}\right] \epsilon_{n} \exp \left(-S\left[\phi_{n}\right]\right)$, where $n$ labels disconnected components of the field configurations. There is no a priori way to fix the relative amplitude $\epsilon_{n}$. When the relative amplitudes between different sectors are complex, this is the physics of topological terms (such as the topological term in the non-linear sigma models) in general. We are thus lead to consider

$$
Z=\sum_{(h, g) \in G^{2}} \epsilon_{g}^{h} \int \mathcal{D}\left[\phi_{g}^{h}\right] e^{-S[\phi]}
$$

where the $\epsilon_{g}^{h}$ are phases that assign different weights to different topological sectors. The phase factors $\epsilon_{g}^{h}$, which are called discrete torsion, can be viewed as arising from projection onto different sectors, or, alternatively, different ways to assign the quantum number for the ground state in each sector.

d. duality between SPT phases and topological phases To summarize, we consider the partition function orbifolded (twisted) by the symmetry group of the problem, and ask if the partition function is modular invariant or not. We view this as a generalization of Laughlin's flux threading argument. It is a generalization in the following two sense: (i) it is a geometrical generalization (ii) symmetry plays an important role. The idea of orbifolding the edge CFT of SPT phases can be viewed in an alternative way, as suggested in Ref 36. (See also Ref 33.) These authors proposed that if the global symmetry of an SPT phase with symmetry group $\mathrm{G}$ is promoted to a gauge symmetry, the resulting phase is a topologically ordered phase with a particular pattern of fractional statistics.

To make a connection with our approach, we first note that the gauging and symmetry projection have a similar effect in that we focus on a gauge singlet sector of the theory [although the gauging means in general imposing singlet condition locally (e.g., at each of a lattice), while projection is enforced only globally] $\underline{48}$ Next, for Abelian theories (multicomponent chiral/nonchiral bosons compactified on a lattice) a self-dual condition together with an even-lattice condition guarantees that modular invariance is achieved. The same condition can be derived from the argument based on fractional statistics as shown in Ref. 33.

\section{ABELIAN BOSONIC SYMMETRY-PROTECTED TOPOLOGICAL PHASES}

In this section, we discuss bosonic SPT phases that can be described in terms of abelian Chern-Simons theories. At the boundary (edge), they support excitations described by a $(1+1)$ dimensional (non-chiral) boson theory. Since it is non-chiral, the edge theory is unstable unless certain discrete symmetry condition $G$ is imposed.

One can view this discrete symmetry as embedded in the $U(1)^{N} \times U(1)^{N}$ continuous symmetry of the Abelian Chern-Simons theory. Within this setting, the gauging can be achieved by the Higgs mechanism. The idea is that by a choice of suitable Higgs fields potentials and couplings one can break the symmetry to the subgroup $G$. One can then study the SPT phase with symmetry group $G$ as a symmetry broken phase of a spontaneously broken gauge symmetry. It is essentially a gauge theory with (discrete) gauge group $G$. The bulk $(2+1)$-dimensional Lagrangian for such a system is given by

$$
\mathcal{L}=\frac{K_{I J}}{4 \pi} \epsilon^{\mu \nu \lambda} a_{\mu}^{I} \partial_{\nu} a_{\lambda}^{J}+q^{I} j_{I}^{\mu} A_{\mu}+\mathcal{L}_{\text {Higgs }}[\{\psi\}, A],
$$

where $K_{I J}$ is a $2 N \times 2 N$ integer valued matrix with $\left|\operatorname{det} K_{I J}\right|=1$. (We shall consider below $N_{f}$ flavors of such theories in which case $K_{I J}$ becomes a block diagonal $2 N N_{f} \times 2 N N_{f}$ matrix). The gauge field $A_{\mu}$ is an external probe field which couples minimally to the internal current $j^{\mu}=\frac{1}{2 \pi} \epsilon^{\mu \nu \lambda} \partial_{\nu} a_{\lambda}$ with the corresponding charge $q_{I}$. The $\{\psi\}$ are Higgs fields chosen in appropriate representations to break the symmetry to $G$. The internal fields $a_{\mu}^{I}$ can be integrated out to obtain an effective Chern-Simons Higgs theory for the external field $A_{\mu}$.

We shall simply consider the edge theory for a bosonic state which for a non-chiral state (after turning off the external potential) can be taken without loss of generality to be $K_{I J}=\sigma_{x}$ ( $\sigma_{x}$ is the $x$ component of the Pauli matrices), by using $G L(2, \mathbb{Z})$ symmetry $K_{I J} \rightarrow W^{T} K_{I J} W$ with $|\operatorname{det} W|=1 .^{29}$ In this case the edge theory is obtianed using a gauge invariance argument of the ChernSimons theory to be

$$
S=\frac{1}{4 \pi} \int d t d x\left(\partial_{t} \phi_{1} \partial_{x} \phi_{2}+\partial_{t} \phi_{2} \partial_{x} \phi_{1}-V_{I J} \partial_{x} \phi_{I} \partial_{x} \phi_{J}\right) \text {. }
$$

(We start with the case of one flavor $N_{f}=1$ and we introduce flavors later).

We consider $G=\mathbb{Z}_{K} \times \mathbb{Z}_{K}$ and consider the symmetry transformation for $\left(k_{1}, l_{1}\right) \in \mathbb{Z}_{K} \times \mathbb{Z}_{K}$

$$
\phi_{1} \rightarrow \phi_{1}+\frac{2 \pi k_{1}}{K}, \quad \phi_{2} \rightarrow \phi_{2}+\frac{2 \pi l_{1}}{K} .
$$


We note that the fields have the periodicity $\phi_{I}=\phi_{I}+2 \pi$ so that this is indeed a $\mathbb{Z}_{K} \times \mathbb{Z}_{K}$ symmetry transformation. Setting $l_{2}=l_{1} q$ for $q \in \mathbb{Z}$ reduces to a $\mathbb{Z}_{K}$ subgroup of $\mathbb{Z}_{K} \times \mathbb{Z}_{K}$ which is one of the cases considered in Ref. 29. We shall analyze the modular invariance properties of the gauged CFT described by the action (10) and symmetry transformation (11).

In terms of the chiral modes $\phi_{L}$ and $\phi_{R}$ defined by

$$
\phi_{1}=\sqrt{\frac{r}{2}}\left(\phi_{L}+\phi_{R}\right), \quad \phi_{2}=\sqrt{\frac{1}{2 r}}\left(\phi_{L}-\phi_{R}\right),
$$

one can rewrite this as

$$
\begin{array}{r}
S=\frac{1}{4 \pi} \int d t d x\left[\partial_{t} \phi_{L} \partial_{x} \phi_{L}-v\left(\partial_{x} \phi_{L}\right)^{2}\right. \\
\left.-\partial_{t} \phi_{R} \partial_{x} \phi_{R}-v\left(\partial_{x} \phi_{R}\right)^{2}\right]
\end{array}
$$

where $r:=\sqrt{\frac{V_{22}}{V_{11}}}$ and we have assumed $V_{12}+V_{21}=0$ so that we obtain a non-chiral theory with equal left and right moving velocities $v=2 \sqrt{V_{11} V_{22}}$. Henceforth, we set $v=1$ and the system size $L=2 \pi v$, without loss of generality. The Hamiltonian and total momentum are given by

$$
\begin{aligned}
H & =\frac{1}{4 \pi} \int d x\left[\left(\partial_{x} \phi_{L}\right)^{2}+\left(\partial_{x} \phi_{R}\right)^{2}\right], \\
P & =\frac{1}{4 \pi} \int d x\left[\left(\partial_{x} \phi_{L}\right)^{2}-\left(\partial_{x} \phi_{R}\right)^{2}\right] .
\end{aligned}
$$

The quantization of the theory is pretty standard. The equal time canonical commutation relations are given by

$$
\begin{aligned}
{\left[\partial_{x} \phi_{L}(x, t), \partial_{y} \phi_{L}(y, t)\right] } & =2 \pi i \partial_{x} \delta(x-y), \\
{\left[\partial_{x} \phi_{R}(x, t), \partial_{y} \phi_{R}(y, t)\right] } & =-2 \pi i \partial_{x} \delta(x-y) .
\end{aligned}
$$

From the equations of motion one can deduce the mode expansions

$$
\begin{aligned}
& \phi_{L}(x, t)=\phi_{L, 0}+p_{L}(t+x)+i \sum_{n \neq 0} \frac{a_{n}}{n} e^{-i n(t+x)}, \\
& \phi_{R}(x, t)=\phi_{R, 0}+p_{R}(t-x)+i \sum_{n \neq 0} \frac{b_{n}}{n} e^{-i n(t-x)},
\end{aligned}
$$

so that Eq. (15) translates into

$$
\begin{array}{r}
{\left[a_{n}, a_{m}\right]=\left[b_{n}, b_{m}\right]=n \delta_{n+m, 0}} \\
{\left[\phi_{R, L}, p_{R, L}\right]=i}
\end{array}
$$

(We will mostly work in the basis where $p_{R, L}$ are diagonal, i.e.,they can be thought of as $c$ numbers. The allowed values of the zero mode $p_{L, R}$ will be determined from the boundary conditions below.) Therefore $a_{n}$ and $b_{n}$ are the oscillator modes of a free boson. The quantum Hamiltonian and total momentum in terms of the oscillators are

$$
\begin{aligned}
H & =\frac{p_{L}^{2}}{2}+\sum_{n>0} a_{-n} a_{n}-\frac{1}{24}+\frac{p_{R}^{2}}{2}+\sum_{n>0} b_{-n} b_{n}-\frac{1}{24} \\
P & =\frac{p_{L}^{2}-p_{R}^{2}}{2}+\sum_{n>0}\left(a_{-n} a_{n}-b_{-n} b_{n}\right) .
\end{aligned}
$$

The constant term in $H$ is due to the regularization of the sum of zero point energies $\sum_{n} n \rightarrow-1 / 12$.

\section{A. $\mathbb{Z}_{K} \times \mathbb{Z}_{K}$ symmetry}

We are interested in gauging the $\mathbb{Z}_{K} \times \mathbb{Z}_{K}$ symmetry (11). Therefore on a torus of modular parameter $\tau$ we need to consider sectors with twisted spatial periodicities

$$
\begin{aligned}
& \phi_{1}(x+2 \pi, t)=\phi_{1}(x, t)+2 \pi\left(n+\frac{k_{1}}{K}\right), \\
& \phi_{2}(x+2 \pi, t)=\phi_{2}(x, t)+2 \pi\left(m+\frac{l_{1}}{K}\right) .
\end{aligned}
$$

where $n, m \in \mathbb{Z}$. In terms of $\phi_{L}$ and $\phi_{R}$, this is

$$
\begin{aligned}
& \phi_{L}(x+2 \pi, t)=\phi_{L}(x, t)+\frac{2 \pi}{\sqrt{2 r}}\left[\left(n+\frac{k_{1}}{K}\right)+r\left(m+\frac{k_{1}}{K}\right)\right], \\
& \phi_{R}(x+2 \pi, t)=\phi_{R}(x, t)+\frac{2 \pi}{\sqrt{2 r}}\left[\left(n+\frac{k_{1}}{K}\right)-r\left(m+\frac{l_{1}}{K}\right)\right] .
\end{aligned}
$$

This leads to the following quantization condition for the zero internal momentum modes

$$
\begin{aligned}
& p_{L}=\frac{1}{\sqrt{2 r}}\left[\left(n+\frac{k_{1}}{K}\right)+r\left(m+\frac{l_{1}}{K}\right)\right], \\
& p_{R}=\frac{1}{\sqrt{2 r}}\left[-\left(n+\frac{k_{1}}{K}\right)+r\left(m+\frac{l_{1}}{K}\right)\right] .
\end{aligned}
$$

We would also need an expression for the operator $\left(\hat{k}_{2}, \hat{l}_{2}\right)$ that implements translations by $\left(k_{2}, l_{2}\right)$ in the Hilbert space. This can be deduced by computing the commutators between the field $\phi_{1,2}$, and the $U(1)$ charges

$$
\begin{aligned}
Q_{1} & :=\frac{1}{2 \pi} \int d y \partial_{y} \phi_{2}(y, t)=\sqrt{\frac{1}{2 r}}\left(p_{L}+p_{R}\right), \\
Q_{2} & :=\frac{1}{2 \pi} \int d y \partial_{y} \phi_{1}(y, t)=\sqrt{\frac{r}{2}}\left(p_{L}-p_{R}\right) .
\end{aligned}
$$

Since $\left[\phi_{1}(x, t), Q_{1}(t)\right]=\left[\phi_{2}(x, t), Q_{2}(t)\right]=i, Q_{I=1,2}$ generates translations of $\phi_{I}$. The desired operator is then given by

$$
\begin{aligned}
\left(\hat{k}_{2}, \hat{l}_{2}\right) & =\exp \frac{2 \pi i}{K}\left[k_{2} Q_{1}+l_{2} Q_{2}\right] \\
& =\exp i\left[\left(m+\frac{l_{1}}{K}\right) \frac{2 \pi k_{2}}{K}+\left(n+\frac{k_{1}}{K}\right) \frac{2 \pi l_{2}}{K}\right]
\end{aligned}
$$

\section{Partition Function}

We are interested in modular properties of the gauged partition function

$$
Z=\frac{1}{K^{2}} \sum_{k_{1}, k_{2}, l_{1}, l_{2}=0}^{K-1} \epsilon_{\left(k_{2}, l_{2}\right)}^{\left(k_{1}, l_{1}\right)} Z_{\left(k_{2}, l_{2}\right)}^{\left(k_{1}, l_{1}\right)}(\tau)
$$


with

$$
Z_{\left(k_{2}, l_{2}\right)}^{\left(k_{1}, l_{1}\right)}=\operatorname{Tr}_{k_{1}, l_{1}}\left[\left(\hat{k}_{2}, \hat{l}_{2}\right) e^{2 \pi i P \tau_{1}-2 \pi \tau_{2} H}\right]
$$

being the partition function in the sector twisted by $\left(k_{1}, l_{1}\right)$ and $\left(k_{2}, l_{2}\right)$ in the spatial and time directions, respectively. Using the previous results (18, 21, 23) one obtains

$$
\begin{aligned}
& Z_{\left(k_{2}, l_{2}\right)}^{\left(k_{1}, l_{1}\right)}(\tau)=|\eta(\tau)|^{-2} \sum_{(n, m) \in \mathbb{Z}^{2}} \\
& \times \exp \left\{\frac{2 \pi i k_{2}}{K}\left(m+\frac{l_{1}}{K}\right)+\frac{2 \pi i l_{2}}{K}\left(n+\frac{k_{1}}{K}\right)\right. \\
& \quad-\pi \tau_{2}\left[\frac{1}{r}\left(n+\frac{k_{1}}{K}\right)^{2}+r\left(m+\frac{l_{1}}{K}\right)^{2}\right] \\
& \left.\quad+2 \pi i \tau_{1}\left(n+\frac{k_{1}}{K}\right)\left(m+\frac{l_{1}}{K}\right)\right\},
\end{aligned}
$$

where $\tau=\tau_{1}+i \tau_{2}$, and $\eta(\tau)$ is the Dedekind eta function which arises as a result of the sum over the modes of the oscillators.

\section{Large gauge anomaly and modular invariance}

Let us explore the behaviour of Eq. (26) under the "large gauge transformations" $k_{1} \rightarrow k_{1} \pm K$ and similarly for $k_{2}, l_{1}$ and $l_{2}$. Under $k_{1} \rightarrow k_{1}+K$ and $l_{1} \rightarrow l_{1}+K$ by relabelling $n \rightarrow n+1$ and $m \rightarrow m+1$ respectively it is easy to see that Eq. (26) is invariant. On the other hand under hand one has the following large gauge anomaly:

$$
\begin{aligned}
& Z_{\left(k_{2} \pm K, l_{2}\right)}^{\left(k_{1}, l_{1}\right)}(\tau)=e^{\frac{ \pm 2 \pi i l_{1}}{K}} Z_{\left(k_{2}, l_{2}\right)}^{\left(k_{1}, l_{1}\right)}(\tau), \\
& Z_{\left(k_{2}, l_{2} \pm K\right)}^{\left(k_{1}, l_{1}\right)}(\tau)=e^{\frac{ \pm 2 \pi i k_{1}}{K}} Z_{\left(k_{2}, l_{2}\right)}^{\left(k_{1}, l_{1}\right)}(\tau) .
\end{aligned}
$$

Under $T$ modular transformations, $\tau \rightarrow \tau+1$, one finds

$$
Z_{\left(k_{2}, l_{2}\right)}^{\left(k_{1}, l_{1}\right)}(\tau+1)=e^{-\frac{2 \pi i k_{1} l_{1}}{K^{2}}} Z_{\left(k_{1}+k_{2}, l_{1}+l_{2}\right)}^{\left(k_{1}, l_{1}\right)}(\tau) .
$$

To determine the behavior under $S$ modular transformations, it is convenient to use the Poisson resummation formula $\sum_{n \in \mathbb{Z}} f(n)=\sum_{n \in \mathbb{Z}} \int_{-\infty}^{\infty} f(x) e^{-2 \pi i x n} d x$ to rewrite Eq. (26) as

$$
\begin{aligned}
Z_{\left(k_{2}, l_{2}\right)}^{\left(k_{1}, l_{1}\right)}(\tau)=|\eta(\tau)|^{-2} \sqrt{\frac{r}{\tau_{2}}} \sum_{(n, m) \in \mathbb{Z}^{2}} \\
\times \exp \left\{\frac{2 \pi i k_{2}}{N}\left(m+\frac{l_{1}}{K}\right)+\frac{2 \pi i k_{1} n}{K}\right. \\
\quad-\frac{\pi r}{\tau_{2}}\left[|\tau|^{2}\left(m+\frac{l_{1}}{K}\right)^{2}+\left(\frac{l_{2}}{K}-n\right)^{2}\right] \\
\left.+\frac{2 \pi r \tau_{1}}{\tau_{2}}\left(n m+\frac{l_{1} n}{K}-\frac{l_{2} m}{K}-\frac{l_{1} l_{2}}{K^{2}}\right)\right\} .
\end{aligned}
$$

Thus, under $S$, i.e., $\tau_{1} \rightarrow-\frac{\tau_{1}}{|\tau|^{2}}, \tau_{2} \rightarrow \frac{\tau_{2}}{|\tau|^{2}}$, by switching $n \rightarrow-m, m \rightarrow n$ one finds

$$
Z_{\left(k_{2}, l_{2}\right)}^{\left(k_{1}, l_{1}\right)}(-1 / \tau)=e^{\frac{2 \pi i\left(l_{1} k_{2}+k_{1} l_{2}\right)}{K^{2}}} Z_{\left(-k_{1},-l_{1}\right)}^{\left(k_{2}, l_{2}\right)}(\tau) .
$$

The total partition function with $N_{f}$ flavors and $\mathbb{Z}_{K} \times$ $\mathbb{Z}_{K}$ gauge invariance is given by

$$
Z(\tau)=\sum_{k_{1}, l_{1}, k_{2}, l_{2}=0}^{K-1} \epsilon_{\left(k_{2}, l_{2}\right)}^{\left(k_{1}, l_{1}\right)}\left[Z_{\left(k_{2}, l_{2}\right)}^{\left(k_{1}, l_{1}\right)}\right]^{N_{f}},
$$

with $\epsilon_{\left(k_{2}, l_{2}\right)}^{\left(k_{1}, l_{1}\right)}$ having the same meaning as before. From Eq. (30) one determines the following conditions for $S$ modular invariance

$$
\begin{aligned}
& \epsilon_{\left(k_{2}, l_{2}\right)}^{\left(k_{1}, l_{1}\right)}=
\end{aligned}
$$

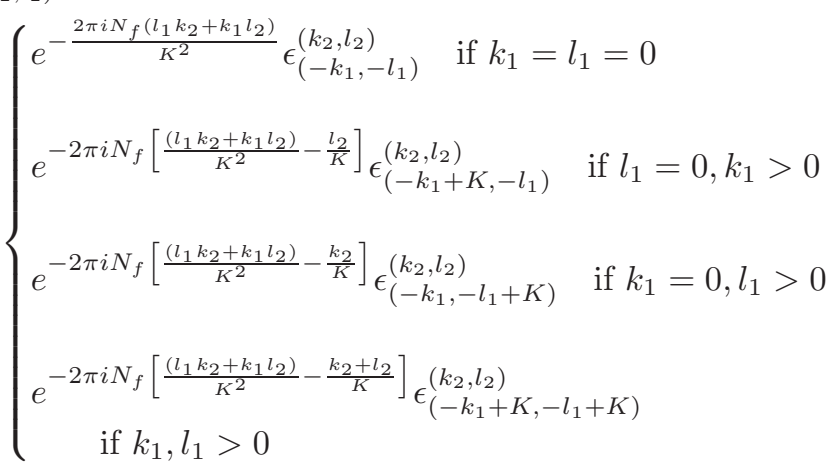

while from (28) one determines the following conditions for $\mathrm{T}$ modular invariance:

$$
\begin{aligned}
& \epsilon_{\left(k_{2}, l_{2}\right)}^{\left(k_{1}, l_{1}\right)}= \\
& \left\{\begin{array}{l}
e^{\frac{2 \pi i N_{f} l_{1} k_{1}}{K^{2}}} \epsilon_{\left(k_{1}+k_{2}, l_{1}+l_{2}\right)}^{\left(k_{1}, l_{1}\right)} \\
\text { if } k_{1}+k_{2}, l_{1}+l_{2} \leq K-1 \\
e^{2 \pi i N_{f}\left[\frac{l_{1} k_{1}}{K^{2}}-\frac{l_{1}}{K}\right]} \epsilon_{\left(k_{1}+k_{2}-K, l_{1}+l_{2}\right)}^{\left(k_{1}, l_{1}\right)} \\
\text { if } k_{1}+k_{2}>K-1, l_{1}+l_{2} \leq K-1 \\
e^{2 \pi i N_{f}\left[\frac{l_{1} k_{1}}{K^{2}}-\frac{k_{1}}{K}\right]} \epsilon_{\left(k_{1}+k_{2}, l_{1}+l_{2}-K\right)}^{\left(k_{1}, l_{1}\right)} \\
\text { if } k_{1}+k_{2} \leq N-1, l_{1}+l_{2}>K-1 \\
e^{2 \pi i N_{f}\left[\frac{l_{1} k_{1}}{K^{2}}-\frac{l_{1}+k_{1}}{K}\right]} \epsilon_{\left(k_{1}+k_{2}-K, l-1+l_{2}-K\right)}^{\left(k_{1}, l_{1}\right)} \\
\text { if } k_{1}+k_{2}, l_{1}+l_{2}>K-1
\end{array}\right.
\end{aligned}
$$

Let us analyze Eqs. (32) and (33) in general. First focus on Eq. (32) and observe that when $K$ is even

$$
\epsilon_{(K / 2, K / 2)}^{(K / 2, K / 2)}=e^{\pi i N_{f}} \epsilon_{(K / 2, K / 2)}^{(K / 2, K / 2)}
$$

so this forces $N_{f}$ be even. For even $K$ this is the only condition required while for odd $K$ there is no condition for consistency of Eq. (32) as is easily verified. 
Now Eq. (33) gives after few iterations

$$
\begin{array}{r}
\epsilon_{(0,0)}^{(1,1)}=e^{\frac{2 \pi i N_{f}}{K^{2}}} \epsilon_{(1,1)}^{(1,1)}=\cdots=e^{\frac{2 \pi i N_{f}(K-1)}{K^{2}}} \epsilon_{(K-1, K-1)}^{(1,1)} \\
=e^{-\frac{2 \pi i N_{f}}{K}} \epsilon_{(0,0)}^{(1,1)},
\end{array}
$$

which is consistent iff $N_{f}=0 \bmod K$.

For generic values of $k_{1}, k_{2}, l_{1}, l_{2}$ one obtains the following consistency condition

$$
\epsilon_{\left(k_{2}, l_{2}\right)}^{\left(k_{1}, l_{1}\right)}=e^{\frac{2 \pi i N_{f} l_{1} k_{1} p}{K^{2}}} \epsilon_{\left(k_{2}, l_{2}\right)}^{\left(k_{1}, l_{1}\right)},
$$

for some integers $p, s, t$ such that $p k_{1}-K t=0$ and $p l_{1}-K s=0$. Therefore $T$ modular invariance is possible iff $N_{f}=0 \bmod K$. It is not difficult to show that with this condition on $N_{f}$ and the phases, $S$ and $T$ modular invariance can be simultaneously achieved. Hence we conclude that modular invariance is possible iff $N_{f}=0$ $\bmod K$.

\section{Example: $\mathbb{Z}_{2} \times \mathbb{Z}_{2}$}

Equation (32) gives $\epsilon_{(1,1)}^{(1,1)}=e^{i N_{f} \pi} \epsilon_{(1,1)}^{(1,1)}$ which is possible, for non-zero $\epsilon_{(1,1)}^{(1,1)}$, iff $N_{f}=0 \bmod 2$. With these conditions (32) and (33) give

$$
\begin{aligned}
& \epsilon_{(0,1)}^{(0,0)}=\epsilon_{(0,0)}^{(0,1)}=\epsilon_{(0,1)}^{(0,1)}, \quad \epsilon_{(1,0)}^{(0,0)}=\epsilon_{(0,0)}^{(1,0)}=\epsilon_{(1,0)}^{(1,0)}, \\
& \epsilon_{(1,1)}^{(0,0)}=\epsilon_{(0,0)}^{(1,1)}=\epsilon_{(1,1)}^{(1,1)}, \\
& \epsilon_{(1,0)}^{(0,1)}=\epsilon_{(1,1)}^{(0,1)}=\epsilon_{(1,0)}^{(1,1)}= \pm \epsilon_{(0,1)}^{(1,1)}= \pm \epsilon_{(0,1)}^{(1,0)}= \pm \epsilon_{(1,1)}^{(1,0)},
\end{aligned}
$$

with the minus signs when $N_{f}=2 \bmod 4$ and plus signs when $N_{f}=0 \bmod 4$. Therefore modular invariance can be achieved iff $N_{f}=0 \bmod 2$. That is the edge theory is expected to be unstable when $N_{f}=0 \bmod 2$. In Sec. IIIC we will construct explicitly a potential that gaps out the edge theory.

\section{B. $\mathbb{Z}_{K}$ symmetry}

To compare our results to that obtained for $\mathbb{Z}_{K}$ SPT phases, $\stackrel{29}{\underline{2}}$ we consider the embedding $\mathbb{Z}_{K} \rightarrow \mathbb{Z}_{K} \times \mathbb{Z}_{K}$ given by $k \rightarrow(k, k q)$ and $N_{f}=1$. To analyze this case we simply set $l_{1}=k_{1} q$ and $l_{2}=k_{2} q$ and $N_{f}=1$ in the previous expressions above. Hence we can write

$$
Z(\tau)=\sum_{k_{1}, k_{2}=0}^{K-1} \epsilon_{k_{2}}^{k_{1}} Z_{k_{2}}^{k_{1}}(\tau)
$$

with $Z_{k_{2}}^{k_{1}}:=Z_{\left(k_{2}, k_{2} q\right)}^{\left(k_{1}, k_{1} q\right)}$ and similarly for $\epsilon$. Therefore in this case the large gauge anomaly is

$$
Z_{k_{2} \pm K}^{k_{1}}=Z_{\left(k_{2} \pm K, k_{2} q \pm q K\right)}^{\left(k_{1}, q k_{1}\right)}=e^{ \pm \frac{4 \pi i q k_{1}}{K}} Z_{k_{2}}^{k_{1}} .
$$

The $T$ modular transformation is now given by

$$
Z_{k_{2}}^{k_{1}}(\tau+1)=e^{-\frac{2 \pi i q k_{1}^{2}}{K^{2}}} Z_{k_{1}+k_{2}}^{k_{1}}(\tau),
$$

while the $S$ modular transformation is

$$
Z_{k_{2}}^{k_{1}}(-1 / \tau)=e^{\frac{4 \pi i q k_{1} k_{2}}{K^{2}}} Z_{-k_{1}}^{k_{2}}(\tau) .
$$

Thus the $S$ modular invariance condition is

$$
\epsilon_{k_{2}}^{k_{1}}=\left\{\begin{array}{l}
e^{-\frac{4 \pi i q k_{1} k_{2}}{K^{2}} \epsilon_{-k_{1}}^{k_{2}}} \quad \text { if } \quad k_{1}=0, \\
e^{-4 \pi i\left(\frac{q k_{1} k_{2}}{K^{2}}-\frac{q k_{2}}{N}\right)} \epsilon_{-k_{1}+K}^{k_{2}} \quad \text { if } \quad k_{1}>0,
\end{array}\right.
$$

while the $T$ modular invariance condition is

$$
\epsilon_{k_{2}}^{k_{1}}=\left\{\begin{array}{lll}
e^{\frac{2 \pi i q k_{1}^{2}}{K^{2}}} \epsilon_{k_{1}+k_{2}}^{k_{1}} & \text { if } & k_{1}+k_{2} \leq K-1, \\
e^{2 \pi i\left[\frac{q k_{1}^{2}}{K^{2}}-\frac{2 q k_{1}}{K}\right]} \epsilon_{k_{1}+k_{2}-K}^{k_{1}} & \text { if } & k_{1}+k_{2}>K-1 .
\end{array}\right.
$$

From Eq. (42) above, one obtains

$$
\epsilon_{k_{2}}^{0}=\epsilon_{0}^{k_{2}}=\epsilon_{-k_{2}+K}^{0}=\epsilon_{0}^{-k_{2}+K},
$$

if $k_{1}=0$ and $k_{2} \neq 0$, whereas

$$
\epsilon_{0}^{k_{1}}=\epsilon_{-k_{1}+K}^{0}=\epsilon_{0}^{-k_{1}+K}=\epsilon_{k_{1}}^{0} .
$$

if $k_{2}=0$ and $k_{1} \neq 0$. Similarly, for even $K$ and $k_{1}=$ $k_{2}=K / 2$ one gets

$$
\epsilon_{K / 2}^{K / 2}=e^{\pi i q} \epsilon_{K / 2}^{K / 2}
$$

which is consistent iff $q=0 \bmod 2$. Finally, for other values of $k_{1}$ and $k_{2}$ different from those considered above one gets

$$
\begin{aligned}
\epsilon_{k_{2}}^{k_{1}} & =e^{-4 \pi i\left(\frac{q k_{1} k_{2}}{K^{2}}-\frac{q k_{2}}{K}\right)} \epsilon_{-k_{1}+K}^{k_{2}} \\
& =e^{-\frac{4 \pi i q k_{1}}{K}} \epsilon_{-k_{2}+K}^{-k_{1}+K} \\
& =e^{-\frac{4 \pi i q k_{1} k_{2}}{K^{2}}} \epsilon_{k_{1}}^{-k_{2}+K}
\end{aligned}
$$

where we have left out a self consistent further iteration. This shows that $S$ modular invariance is possible for even $K$ iff $q$ is even and for odd $K$ there is no condition on $q$.

Now we move on to analyze the $T$ modular invariance conditions which imply

$$
\epsilon_{0}^{1}=e^{\frac{2 \pi i q}{K^{2}}} \epsilon_{1}^{1}=\cdots=e^{\frac{2 \pi i q(K-1)}{K^{2}}} \epsilon_{K-1}^{1}=e^{-\frac{2 \pi i q}{K}} \epsilon_{0}^{1},
$$

which is self consistent iff $q=0 \bmod K$. We now show that this condition is sufficient for $T$ modular invariance. When $k_{1} \neq 0$ after several reiterations one gets the condition

$$
\epsilon_{k_{2}}^{k_{1}}=e^{\frac{2 \pi i q k_{1}^{2} p}{K^{2}}} \epsilon_{k_{2}}^{k_{1}},
$$

where $p$ is an integer such that $k_{1} p-K t=0$ for an integer $t$ whose actual value is irrelevant to us. So that with $q=0 \bmod K$ the phase in Eq. (49) is just 1.

Thus putting our results together we find that modular invariance is possible iff $q=0 \bmod K$. I.e., the phase is trivial when $q=0 \bmod K$ and non-trivial (i.e., SPT phase) otherwise. 


\section{Gapping potential perspective}

In this section we show that there exist potentials that can fully gap our system without explicitly or spontaneously breaking the $\mathbb{Z}_{2} \times \mathbb{Z}_{2}$ symmetry iff $N_{f}=0 \bmod$ 2. Thus we confirm that we do indeed have a SPT phase when $N_{f} \neq 0 \bmod 2$ and a trivial phase otherwise.

Let us first consider the case when $N_{f}=1$, in this case a complete set of local operators in the field theory is given by $\partial \Phi, \partial \Theta$, and $\cos (m \Phi+n \Theta+\alpha)$, where $\partial$ denotes a generic derivative and $\alpha$ is a constant. Here we have switched our notation, $\phi_{1} \rightarrow \Phi$ and $\phi_{2} \rightarrow \Theta$, to emphasize the dual (canonical conjugate) nature of these fields, $[\Phi, \partial \Theta] \sim 2 \pi i$. The most general gapping potential terms that is $\mathbb{Z}_{2} \times \mathbb{Z}_{2}$ symmetric is given by linear combinations of the form

$$
\cos (2 m \Phi+2 n \Theta+\alpha)
$$

Now we can do a semi-classical analysis to show that the ground state spontaneously breaks $\mathbb{Z}_{2} \times \mathbb{Z}_{2}$ symmetry once a strong enough gapping potential of the form (50) is added. Without loss of generality we set $\alpha=0$, and since $[\Phi, \partial \Theta] \sim 2 \pi i$ etc., we need to consider independently potentials of the form $\cos (2 m \Phi)$ and $\cos (2 n \Theta)$. For $\cos (2 m \Phi)$ we have that classical minima correspond to $\Phi=\frac{2 j+1}{2 m} \pi$ (for a finite number of independent $j$ 's since $\Phi \in[0,2 \pi))$ and the $\mathbb{Z}_{2}$ transformation $\Phi \rightarrow \Phi+\pi$ amounts to $j \rightarrow j+m$. As one can easily see when $m=1$, for example, these classical minima transform under $\mathbb{Z}_{2}$ and a choice of any one of them would spontaneously break $\mathbb{Z}_{2}$ symmetry. The analysis for potentials of the form $\cos (2 n \Theta)$ is similar and we reach the conclusion that when $N_{f}=1$ it is not possible to gap our system without breaking $\mathbb{Z}_{2} \times \mathbb{Z}_{2}$ symmetry.

When $N_{f}=2$ we have fields $\Phi_{1}, \Theta_{1}, \Phi_{2}, \Theta_{2}$ and the most general $\left(\mathbb{Z}_{2} \times \mathbb{Z}_{2}\right)$-invariant possible gapping potential is given by $\cos \left[m_{1}\left(\Phi_{1}-\Phi_{2}\right)+n_{1}\left(\Theta_{1}-\Theta_{2}\right)+\right.$ $\left.2 l_{1} \Phi_{1}+2 l_{2} \Theta_{2}+\alpha\right]$ where $m_{1}, n_{1}, l_{1}, l_{2} \in \mathbb{Z}$. If we focus on two mutually commuting and $\left(\mathbb{Z}_{2} \times \mathbb{Z}_{2}\right)$-symmetric bosonic fields $\Phi_{1}-\Phi_{2}$ we can consider a gapping potential $\cos \left(m_{1}\left(\Phi_{1}-\Phi_{2}\right)+n_{1}\left(\Theta_{1}+\Theta_{2}\right)\right)$. If we consider $-\cos \left(\Phi_{1}-\Phi_{2}\right)-\cos \left(\Theta_{1}+\Theta_{2}\right)$ we see that the classical minima corresponds to

$$
\Phi_{1}-\Phi_{2}=0, \quad \Theta_{1}+\Theta_{2}=2 \pi,
$$

since this is invariant under $\mathbb{Z}_{2} \times \mathbb{Z}_{2}$ and $\left[\Phi_{1}-\Phi_{2}, \Theta_{1}+\right.$ $\left.\Theta_{2}\right]=0$ we conclude that we can fully gap the system without spontaneously breaking the symmetry.

Most generally when $N_{f}=2 m$ we have fields $\Phi_{i}, \Theta_{i}$ $(i=1, \ldots, 2 m)$ and the most general $\left(\mathbb{Z}_{2} \times \mathbb{Z}_{2}\right)$ invariant possible gapping potential is given by a sum over potentials of the form

$$
\begin{array}{r}
\cos \left\{\sum_{i=1}^{2 m-1} m_{i}\left(\Phi_{i}-\Phi_{2 m}\right)+\sum_{i=1}^{2 m-1} n_{i}\left(\Theta_{i}-\Theta_{2 m}\right)\right. \\
\left.+2 l_{1} \Phi_{2 m}+2 l_{2} \Theta_{2 m}+\alpha\right\} .
\end{array}
$$

If we focus on two mutually commuting and $\left(\mathbb{Z}_{2} \times \mathbb{Z}_{2}\right)$ symmetric bosonic fields then a potential of the form

$$
-\sum_{i=1}^{m}\left[\cos \left(\Phi_{2 i-1}-\Phi_{2 i}\right)+\cos \left(\Theta_{2 i-1}+\Theta_{2 i}\right)\right]
$$

would be minimized classically by

$$
\Phi_{2 i-1}-\Phi_{2 i}=0, \quad \Theta_{2 i-1}+\Theta_{2 i}=2 \pi
$$

which is allowed since all the commutators between the different fields on the left-hand side (LHS) of (54) are zero. On the other hand when $M=2 m+1$, by choosing a maximal set of $2 m$ commuting $\left(\mathbb{Z}_{2} \times \mathbb{Z}_{2}\right)$-invariant fields (basically differences $\Phi_{i}-\Phi_{j}$ etc) to localize, one is always left with one field that cannot be localized and hence the system cannot be fully gapped. This concludes our analysis of bosonic SPT phases with $\mathbb{Z}_{2} \times \mathbb{Z}_{2}$ symmetry.

For general $K$ the above analysis is easily generalized. For example, for $K=3$ with $N_{f}=3$ one can gap out the following independent, mutually commuting, and $\left(\mathbb{Z}_{K} \times\right.$ $\mathbb{Z}_{K}$ )-invariant combinations

$$
\Phi_{1}-\Phi_{2}, \quad \Phi_{2}-\Phi_{3}, \quad \Theta_{1}+\Theta_{2}+\Theta_{3},
$$

while for $K=4$ with $N_{f}=4$ one can gap out

$$
\begin{array}{ll}
\Phi_{1}-\Phi_{2}, & \Theta_{1}+\Theta_{2}-\Theta_{3}-\Theta_{4} \\
\Phi_{3}-\Phi_{4}, & \Theta_{1}+\Theta_{2}+\Theta_{3}+\Theta_{4} .
\end{array}
$$

For generic $K$, one could consider, for example, a gapping potential $\sum_{i=1}^{K-1} \cos \left[m_{i}\left(\Phi_{i}-\Phi_{i-1}\right)\right]+\cos \left[n \sum_{i}^{K} \Theta_{i}\right]$ that gaps $K=N_{f}$ mutually independent and $\left(\mathbb{Z}_{K} \times \mathbb{Z}_{K}\right)$ symmetric combinations of bosonic fields.

\section{FERMIONIC SYMMETRY PROTECTED TOPOLOGICAL PHASES}

In this section, we are interested in fermionic SPT phases which are relevant to the physics of topological superconductors. We consider $\mathbb{Z}_{K} \times \mathbb{Z}_{K}$ symmetry which is a generalization of the $\mathbb{Z}_{2} \times \mathbb{Z}_{2}$ symmetry discussed in Refs. 38 and 44 . The relevant edge theory in the absence of interactions with several flavors is described by the free Dirac action

$S=\frac{i}{2 \pi} \int d x d t \sum_{a=1}^{N_{f}} \psi_{L a}^{\dagger}\left(\partial_{t}-\partial_{x}\right) \psi_{L a}+\psi_{R a}^{\dagger}\left(\partial_{t}+\partial_{x}\right) \psi_{R a}$

where $\psi_{L}^{\dagger}, \psi_{L}, \psi_{R}^{\dagger}, \psi_{R}$ are creation/annihilation operators of the fermionic nonchiral edge modes that are supported by some topological bulk system. This action has a $U(1) \times U(1)$ symmetry, which contains $\mathbb{Z}_{K} \times \mathbb{Z}_{K}$ as its subgroup. As in the bosonic case we gauge this $\mathbb{Z}_{K} \times \mathbb{Z}_{K}$ subgroup to understand the corresponding SPT phases. 


\section{A. Partition function and modular transformation}

The Hamiltonian $H$ and momentum $P$, when $N_{f}=1$, are

$$
\begin{aligned}
& H=\frac{i}{2 \pi} \int d x\left[\psi_{L}^{\dagger} \partial_{x} \psi_{L}-\psi_{R}^{\dagger} \partial_{x} \psi_{R}\right] \\
& P=\frac{i}{2 \pi} \int d x\left[\psi_{L}^{\dagger} \partial_{x} \psi_{L}+\psi_{R}^{\dagger} \partial_{x} \psi_{R}\right]
\end{aligned}
$$

There is an obvious global $U(1) \times U(1)$ symmetry under the transformations

$$
\psi_{L} \rightarrow e^{2 \pi i \alpha} \psi_{L}, \quad \psi_{R} \rightarrow e^{2 \pi i \tilde{\alpha}} \psi_{R}
$$

with the left movers neutral under the second $U(1)$ factor and the right movers neutral under the first $U(1)$ factor. This is generated by the left and right "fermion number" charges

$$
Q_{L}=\frac{1}{2 \pi} \int d x \psi_{L}^{\dagger} \psi_{L}, \quad Q_{R}=\frac{1}{2 \pi} \int d x \psi_{R}^{\dagger} \psi_{R}
$$

The total fermion number is $Q:=Q_{L}+Q_{R}$. We work on a complex torus of modulus $\tau$ and consider sectors with the following periodicity conditions:

$$
\begin{aligned}
\psi_{L}(x+2 \pi, t) & =e^{-2 \pi i \alpha} \psi_{L}(x, t), \\
\psi_{L}\left(x+2 \pi \tau_{1}, t+2 \pi \tau_{2}\right) & =e^{-2 \pi i \beta} \psi_{L}(x, t), \\
\psi_{R}(x+2 \pi, t) & =e^{2 \pi i \tilde{\alpha}} \psi_{R}(x, t), \\
\psi_{R}\left(x+2 \pi \tau_{1}, t+2 \pi \tau_{2}\right) & =e^{2 \pi i \tilde{\beta}} \psi_{R}(x, t) .
\end{aligned}
$$

(In the operator formalism, we can account for the spatial periodicity by appropriate mode expansions and for the time periodicity by insertion of an appropriate operator.) The mode expansions are

$$
\begin{aligned}
& \psi_{L}(x, t)=\sum_{r \in \mathbb{Z}+\alpha} a_{r} e^{-i r(x+t)}, \\
& \psi_{R}(x, t)=\sum_{r \in \mathbb{Z}+\tilde{\alpha}} b_{r} e^{-i r(t-x)},
\end{aligned}
$$

with anticommutators

$$
\left\{a_{r}, a_{s}^{\dagger}\right\}=\left\{b_{r}, b_{s}^{\dagger}\right\}=\delta_{r, s} .
$$

We introduce the normal ordering with respect to the fermionic ground state as

$$
: a_{r}^{\dagger} a_{r}:= \begin{cases}a_{r}^{\dagger} a_{r} & \text { if } r \geq 0 \\ -a_{r} a_{r}^{\dagger} & \text { if } r<0\end{cases}
$$

and similarly for the $b$ oscillators. So the left-moving contribution to $H, H_{L}=\sum_{r \in \mathbb{Z}+\alpha} r a_{r}^{\dagger} a_{r}$, is now given by

$$
H_{L}=\sum_{r \in \mathbb{Z}+\alpha} r: a_{r}^{\dagger} a_{r}:-\frac{1}{24}+\frac{1}{2}\left(\alpha-[\alpha]-\frac{1}{2}\right)^{2},
$$

where we have adopted the regularization prescription $\sum_{r<0} r=E_{0}(\alpha)=-1 / 24+(1 / 2)(\alpha-[\alpha]-1 / 2)^{2}$ and $[\alpha]$ is the greatest integer less than or equal to $\alpha$. We get a similar contribution from the right movers with $\alpha$ replaced by $\tilde{\alpha}, H_{R}=\sum_{s \in \mathbb{Z}+\tilde{\alpha}} s b_{s}^{\dagger} b_{s}$. The left moving contribution to the fermion number, $Q_{L}=\sum_{r \in \mathbb{Z}+\alpha} a_{r}^{\dagger} a_{r}$, is

$$
Q_{L}=\sum_{r}: a_{r}^{\dagger} a_{r}:+\alpha-[\alpha]-\frac{1}{2}
$$

with the regularization prescription $\sum_{r<0} 1=\alpha-[\alpha]-$ $1 / 2$. We get a similar contribution from the right movers with $\alpha$ replaced by $\tilde{\alpha}, Q_{R}=\sum_{s \in \mathbb{Z}+\tilde{\alpha}} b_{s}^{\dagger} b_{s}$.

We would like to evaluate the partition function in Euclidean signature for fermions with boundary conditions (61). This is given by $\left(q:=e^{2 \pi i \tau}\right)$

$$
\begin{aligned}
& Z_{\beta \tilde{\beta}}^{\alpha \tilde{\alpha}}(\tau)=\operatorname{Tr}_{\alpha}\left[e^{2 \pi i Q_{L}\left(-\beta+\frac{1}{2}\right)} q^{H_{L}}\right] \\
& \quad \times \operatorname{Tr}_{\tilde{\alpha}}\left[e^{-2 \pi i Q_{R}\left(-\tilde{\beta}+\frac{1}{2}\right)} \bar{q}^{H_{R}}\right]=Z_{\beta}^{\alpha}(\tau) \bar{Z}_{\tilde{\beta}}^{\tilde{\alpha}}(\tau),
\end{aligned}
$$

where the trace is taken over the fermionic Fock space generated by the fermonic oscillator modes in (62) acting on the Dirac sea. So the partition function has a left-right factorized form. We focus on the left movers since the analysis is similar for the right movers.

$$
\begin{aligned}
& Z_{\beta}^{\alpha}(\tau) \\
= & \operatorname{Tr}_{\alpha}\left[e^{2 \pi i\left(\sum_{r}: a_{r}^{\dagger} a_{r}:+\alpha-[\alpha]-\frac{1}{2}\right)\left(-\beta+\frac{1}{2}\right)} q^{\sum_{r} r: a_{r}^{\dagger} a_{r}:+E_{0}(\alpha)}\right] \\
= & e^{2 \pi i\left(\alpha-[\alpha]-\frac{1}{2}\right)\left(-\beta+\frac{1}{2}\right)} \prod_{r \geq 0}\left[1+e^{2 \pi i\left(-\beta+\frac{1}{2}\right)} q^{r}\right] \\
& \times q^{E_{0}(\alpha)} \prod_{r<0}\left[1+e^{-2 \pi i\left(-\beta+\frac{1}{2}\right)} q^{-r}\right] .
\end{aligned}
$$

Note that $Z_{\beta}^{\alpha+1}(\tau)=Z_{\beta}^{\alpha}(\tau)$ and $Z_{\beta+1}^{\alpha}(\tau)=$ $e^{-2 \pi i\left(\alpha-[\alpha]-\frac{1}{2}\right)} Z_{\beta}^{\alpha}(\tau)$. We can write the partition function as

$$
\begin{aligned}
Z_{\beta}^{\alpha}(\tau)= & e^{\pi i \tau\left([\alpha]^{2}-2\left(\alpha-\frac{1}{2}\right)[\alpha]\right)-2 \pi i[\alpha]\left(-\beta+\frac{1}{2}\right)} \\
& \times \frac{\theta\left[\begin{array}{c}
\alpha-\frac{1}{2} \\
-\beta+\frac{1}{2}
\end{array}\right](0, \tau)}{\eta(\tau)}
\end{aligned}
$$

where the theta function with characteristics is given by

$$
\theta\left[\begin{array}{l}
a \\
b
\end{array}\right](\nu, \tau)=\sum_{n \in \mathbb{Z}} e^{\pi i(n+a)^{2} \tau+2 \pi i(n+a)(\nu+b)}
$$

If we choose $\alpha \in[0,1)$ then $[\alpha]=0$ and the phase factor in Eq. (69) cancels.

By noting

$$
\theta\left[\begin{array}{l}
a \\
b
\end{array}\right](\nu, \tau+1)=e^{-\pi i\left(a^{2}+a\right)} \theta\left[\begin{array}{c}
a \\
b+a+\frac{1}{2}
\end{array}\right](\nu, \tau)
$$


and using the Poisson resummation formula one finds

$$
\theta\left[\begin{array}{l}
a \\
b
\end{array}\right]\left(\nu,-\frac{1}{\tau}\right)=(-i \tau)^{\frac{1}{2}} e^{2 \pi i a(\nu+b)} \theta\left[\begin{array}{c}
-\nu-b \\
a
\end{array}\right](0, \tau) .
$$

From these one deduces the following modular transformations of the right moving contribution to the partition function:

$$
\begin{aligned}
& Z_{\beta}^{\alpha}(\tau+1)=e^{-\pi i\left(\alpha^{2}-\frac{1}{6}\right)} Z_{\beta-\alpha}^{\alpha}(\tau), \\
& Z_{\beta}^{\alpha}(-1 / \tau)=e^{2 \pi i\left(\alpha-\frac{1}{2}\right)\left(-\beta+\frac{1}{2}\right)} Z_{1-\alpha}^{\beta}(\tau),
\end{aligned}
$$

where we have assumed $\alpha, \beta \in[0,1)$ as would be the relevant case in what follows.

\section{B. $\mathbb{Z}_{K} \times \mathbb{Z}_{K}$ symmetry}

We are interested in gauging $\mathbb{Z}_{K} \times \mathbb{Z}_{K}$ symmetry of $N_{f}$ Dirac fermions ( $2 N_{f}$ Majorana fermions) which is generated by

$$
\psi_{L}^{a} \rightarrow e^{-\frac{2 \pi i}{K}} \psi_{L}^{a} \quad \text { and } \quad \psi_{R}^{a} \rightarrow e^{\frac{2 \pi i}{K}} \psi_{R}^{a}
$$

where $a=1, \ldots, N_{f}$. Thus we shall be analyzing the modular properties of the most general partition function

$$
Z(\tau)=\sum_{k, l, m, n} \epsilon_{(k, l, m, n)}\left[Z_{l^{\prime}}^{k^{\prime}}(\tau) \bar{Z}_{n^{\prime}}^{m^{\prime}}(\tau)\right]^{N_{f}},
$$

with $k, l, m, n=0,1, \ldots, K-1$, and $k^{\prime}:=k / K$ etc. This corresponds to a sum over the most general combination of left and right twistings of the spinors by the $\mathbb{Z}_{K} \times \mathbb{Z}_{K}$ action. With the $\epsilon_{(k, l, m, n)}$ phases, topologically distinct sectors in the path integral sum are weighted differently. The modular transformations are given by

$$
\begin{aligned}
Z(\tau+1)= & \sum_{k, l, m, n} \epsilon_{(k, l, m, n)} e^{-\pi i N_{f}\left(\frac{k^{2}-m^{2}}{K^{2}}\right)} \\
& \times\left[Z_{l^{\prime}-k^{\prime}}^{k^{\prime}}(\tau) \bar{Z}_{n^{\prime}-m^{\prime}}^{m^{\prime}}(\tau)\right]^{N_{f}} \\
Z(-1 / \tau)= & \sum_{k, l, m, n} \epsilon_{(k, l, m, n)} e^{2 \pi i N_{f}\left(\frac{m n-k l}{K^{2}}+\frac{k-m+n-l}{2 K}\right)} \\
& \times\left[Z_{1-k^{\prime}}^{l^{\prime}}(\tau) \bar{Z}_{1-m^{\prime}}^{n^{\prime}}(\tau)\right]^{N_{f}}
\end{aligned}
$$

As in the bosonic case, we will ask when the modular invariance can be achieved for different values of $K$ and $N_{f}$. Instead of being exhaustive, we make an ansatz that the overall partition function is holomorphically factorized so that

$$
Z(\tau)=Z_{L}(\tau) \bar{Z}_{R}(\tau)=\left|Z_{L}(\tau)\right|^{2} .
$$

In this case modular invariance is achieved as long as the left(right)-moving contribution transforms covariantly with a phase under a generic modular transformation $U, Z_{L}(U \tau)=e^{i \theta} Z_{L}(\tau)$. So we can focus on the left-moving sector,

$$
Z_{L}(\tau)=\sum_{k, l=0}^{K-1} \epsilon_{l}^{k} Z_{l^{\prime}}^{k^{\prime}}(\tau) .
$$

The condition for $S$ modular invariance is then deduced from Eq. (77) to be (the modular invariance conditions presented below are all up to an overall phase)

$$
\epsilon_{l}^{k}= \begin{cases}e^{2 \pi i N_{f}\left(\frac{k l}{K^{2}}+\frac{l-k}{2 K}\right)} \epsilon_{K-k}^{l} & \text { if } k>0 \\ e^{2 \pi i N_{f}\left(\frac{3 l}{2 K}\right)} \epsilon_{-k}^{l} & \text { if } k=0\end{cases}
$$

while from (76) the conditions for $T$ modular invariance is found to be

$$
\epsilon_{l}^{k}= \begin{cases}e^{\frac{\pi i N_{f} k^{2}}{K^{2}}} \epsilon_{l-k}^{k} & \text { if } 0 \leq l-k \leq K-1 \\ e^{2 \pi i N_{f}\left[\frac{k^{2}}{2 K^{2}}+\frac{1}{2}-\frac{k}{K}\right]} \epsilon_{l-k+K}^{k} & \text { if } l-k<0\end{cases}
$$

Let us analyze Eq. (80) in general. When $K$ is even one gets

$$
\epsilon_{k / 2}^{k / 2}=e^{\pi i N_{f} / 2} \epsilon_{k / 2}^{k / 2}
$$

so this requires $N_{f}=0 \bmod 4$. One can check that this condition is enough for self consistency of Eq. (80) while for odd $K$, no condition on $N_{f}$ is required. Equation (81) gives after $K$ iterations

$$
\epsilon_{0}^{1}=e^{\pi i N_{f} \frac{K-1}{K}} \epsilon_{0}^{1} .
$$

When $K$ is even this requires $N_{f}=0 \bmod 2 K$ while for odd $K$ this requires $N_{f}=0 \bmod K$. In general several iterations of Eq. (81) gives

$$
\epsilon_{l}^{k}=e^{\pi i N_{f}\left(\frac{k^{2} p}{K^{2}}+t-\frac{2 k t}{K}\right)} \epsilon_{l}^{k},
$$

for some integers $p$ and $t$ such that

$$
K t-k p=0 .
$$

For even $K$ one sees that $N_{f}=0 \bmod 2 K$ is sufficient while for odd $K$ with $N_{f}=0 \bmod K$ the phase in (84) is $e^{\pi i N_{f} t \frac{k+K}{K}}$. So one needs to consider only the case when $k$ is even. In this case (85) implies that $t$ must be even. Hence the phase is one and we conclude that modular invariance is possible for even $K$ iff $N_{f}=0 \bmod 2 K$ while for odd $K$ iff $N_{f}=0 \bmod K$.

\section{C. $\mathbb{Z}_{2} \times \mathbb{Z}_{2}$ Example}

For the case $\mathbb{Z}_{2} \times \mathbb{Z}_{2}$, let us check that we recover the results in Ref. 38 from our analysis. In this case Eq. (80) gives

$$
\epsilon_{1}^{0}=e^{\frac{3 \pi i N_{f}}{2}} \epsilon_{0}^{1}, \quad \epsilon_{0}^{1}=e^{-\frac{\pi i N_{f}}{2}} \epsilon_{1}^{0}, \quad \epsilon_{1}^{1}=e^{\frac{\pi i N_{f}}{2}} \epsilon_{1}^{1},
$$


while Eq. (81) gives

$$
\epsilon_{0}^{1}=e^{-\frac{3 \pi i N_{f}}{4}} \epsilon_{1}^{1}, \quad \epsilon_{1}^{1}=e^{\frac{\pi i N_{f}}{4}} \epsilon_{0}^{1} .
$$

Clearly Eqs. (86) and (87) are consistent iff $N_{f}=0 \bmod$ 4 which recovers the result in Ref. 38 with $N_{f}=2 N$ being the number of flavors of Majorana modes. This result is, of course, familiar from type II string theory 50 where the $\mathbb{Z}_{2}$ symmetry is generated by the fermion number current on the world sheet and the Majorana modes corresponds to directions in spacetime with two extra dimensions cancelling the ghosts modes that result from gauge fixing. Thus the GSO left right assymmetric $\mathbb{Z}_{2}$ projection gives rise to consistent modular invariant superstring theories in $8+2$ spacetime dimensions.

\section{Gapping potential perspective}

To analyze the stability to interactions it is convenient to bosonize the fermionic fields as follows:

$$
\psi_{L}=A e^{i \phi_{L}}, \quad \psi_{R}=A e^{i \phi_{R}},
$$

where an implicit normal ordering has been omitted on the right hand side. We would also make use of

$$
2 \phi_{L}=\phi+\theta, \quad 2 \phi_{R}=\phi-\theta,
$$

so that the $\mathbb{Z}_{K} \times \mathbb{Z}_{K}$ symmetry is generated by

$$
\phi_{L} \rightarrow \phi_{L}-\frac{2 \pi}{K}, \quad \phi_{R} \rightarrow \phi_{R}+\frac{2 \pi}{K} .
$$

Let us first consider the simplest case of $\mathbb{Z}_{2} \times \mathbb{Z}_{2}$. With four flavors one can write down the interaction term

$$
\begin{array}{r}
V_{2}=\left(\psi_{R, 1}^{\dagger} \psi_{R, 2}^{\dagger} \psi_{L, 3}^{\dagger} \psi_{L, 4}^{\dagger}+\psi_{R, 1}^{\dagger} \psi_{R, 3}^{\dagger} \psi_{L, 2}^{\dagger} \psi_{L, 4}^{\dagger}\right. \\
\left.+\psi_{R, 1}^{\dagger} \psi_{R, 4}^{\dagger} \psi_{L, 2}^{\dagger} \psi_{L, 3}^{\dagger}+L \leftrightarrow R\right)+ \text { c.c. }
\end{array}
$$

In the bosonized form this is just

$$
V_{2}=4 A^{4} \cos \tilde{\phi}_{1}\left(\cos \tilde{\theta}_{2}+\cos \tilde{\theta}_{3}+\cos \tilde{\theta}_{4}\right)
$$

where

$$
\begin{aligned}
& 2 \tilde{\phi}_{1}=\phi_{1}+\phi_{2}+\phi_{3}+\phi_{4}, \\
& 2 \tilde{\theta}_{2}=\theta_{1}+\theta_{2}-\theta_{3}-\theta_{4}, \\
& 2 \tilde{\theta}_{3}=\theta_{1}+\theta_{3}-\theta_{2}-\theta_{4}, \\
& 2 \tilde{\theta}_{4}=\theta_{1}+\theta_{4}-\theta_{2}-\theta_{3},
\end{aligned}
$$

using $\left[\phi_{i}, \theta_{j}\right] \sim i \delta_{i j}$ one sees that all the fields in Eq. (93) mutually commute and can be simultaneously localized. Also one sees that there are two solutions for the classical minima given by

$$
\tilde{\phi}_{1}=\pi m_{1}, \quad \tilde{\theta}_{2}=\pi n_{2}, \quad \tilde{\theta}_{3}=\pi n_{3}, \quad \tilde{\theta}_{4}=\pi n_{4},
$$

with either $m_{1}$ odd and $n_{2}, n_{3}, n_{4}$ even or $m_{1}$ even and $n_{2}, n_{3}, n_{4}$ odd. By using the periodicity $\phi_{i}=\phi_{i}+2 \pi$ and similarly for $\theta_{i}$ one sees that these two solutions are equivalent and the invariance of $\tilde{\phi}_{1}, \tilde{\theta}_{2}, \tilde{\theta}_{3}, \tilde{\theta}_{4}$ implies that the system can be fully gapped without explicitly or spontaneously breaking $\mathbb{Z}_{2} \times \mathbb{Z}_{2}$ symmetry.

Let us now consider the case of $\mathbb{Z}_{4} \times \mathbb{Z}_{4}$ symmetry as this would enable us to understand the generalization to $\mathbb{Z}_{2 K} \times \mathbb{Z}_{2 K}$. In this case with $N_{f}=8$ one can write down the following potential

$$
\begin{aligned}
V_{4}= & \psi_{R, 1}^{\dagger} \psi_{R, 2}^{\dagger} \psi_{R, 3}^{\dagger} \psi_{R, 4}^{\dagger} \psi_{L, 5}^{\dagger} \psi_{L, 6}^{\dagger} \psi_{L, 7}^{\dagger} \psi_{L, 8}^{\dagger} \\
& +\psi_{R, 1}^{\dagger} \psi_{R, 2}^{\dagger} \psi_{R, 6}^{\dagger} \psi_{R, 8}^{\dagger} \psi_{L, 3}^{\dagger} \psi_{L, 4}^{\dagger} \psi_{L, 5}^{\dagger} \psi_{L, 7}^{\dagger} \\
& +\psi_{R, 1}^{\dagger} \psi_{R, 2}^{\dagger} \psi_{R, 5}^{\dagger} \psi_{R, 6}^{\dagger} \psi_{L, 3}^{\dagger} \psi_{L, 4}^{\dagger} \psi_{L, 7}^{\dagger} \psi_{L, 8}^{\dagger} \\
& +\psi_{R, 1}^{\dagger} \psi_{R, 3}^{\dagger} \psi_{R, 5}^{\dagger} \psi_{R, 7}^{\dagger} \psi_{L, 2}^{\dagger} \psi_{L, 4}^{\dagger} \psi_{L, 6}^{\dagger} \psi_{L, 8}^{\dagger} \\
& +\psi_{R, 1}^{\dagger} \psi_{R, 3}^{\dagger} \psi_{R, 6}^{\dagger} \psi_{R, 8}^{\dagger} \psi_{L, 2}^{\dagger} \psi_{L, 4}^{\dagger} \psi_{L, 5}^{\dagger} \psi_{L, 7}^{\dagger} \\
& +\psi_{R, 1}^{\dagger} \psi_{R, 4}^{\dagger} \psi_{R, 5}^{\dagger} \psi_{R, 8}^{\dagger} \psi_{L, 2}^{\dagger} \psi_{L, 3}^{\dagger} \psi_{L, 6}^{\dagger} \psi_{L, 7}^{\dagger} \\
& +\psi_{R, 1}^{\dagger} \psi_{R, 4}^{\dagger} \psi_{R, 6}^{\dagger} \psi_{R, 7}^{\dagger} \psi_{L, 2}^{\dagger} \psi_{L, 3}^{\dagger} \psi_{L, 5}^{\dagger} \psi_{L, 8}^{\dagger} .
\end{aligned}
$$

In terms of bosonized fields this is

$$
V_{4}=4 A^{8} \cos \tilde{\phi}_{1}\left(\sum_{i=2}^{8} \cos \tilde{\theta}_{i}\right)
$$

where

$$
\begin{aligned}
& 2 \tilde{\phi}_{1}=\sum_{i=1}^{8} \phi_{i}, \\
& 2 \tilde{\theta}_{2}=\theta_{1}+\theta_{2}+\theta_{3}+\theta_{4}-\theta_{5}-\theta_{6}-\theta_{7}-\theta_{8}, \\
& 2 \tilde{\theta}_{3}=\theta_{1}+\theta_{2}+\theta_{6}+\theta_{8}-\theta_{3}-\theta_{4}-\theta_{5}-\theta_{7}, \\
& 2 \tilde{\theta}_{4}=\theta_{1}+\theta_{2}+\theta_{5}+\theta_{6}-\theta_{3}-\theta_{4}-\theta_{7}-\theta_{8}, \\
& 2 \tilde{\theta}_{5}=\theta_{1}+\theta_{3}+\theta_{5}+\theta_{7}-\theta_{2}-\theta_{4}-\theta_{6}-\theta_{8}, \\
& 2 \tilde{\theta}_{6}=\theta_{1}+\theta_{3}+\theta_{6}+\theta_{8}-\theta_{2}-\theta_{4}-\theta_{5}-\theta_{7}, \\
& 2 \tilde{\theta}_{7}=\theta_{1}+\theta_{4}+\theta_{5}+\theta_{8}-\theta_{2}-\theta_{3}-\theta_{6}-\theta_{7}, \\
& 2 \tilde{\theta}_{8}=\theta_{1}+\theta_{4}+\theta_{6}+\theta_{7}-\theta_{2}-\theta_{3}-\theta_{5}-\theta_{8},
\end{aligned}
$$

since the tilded fields are mutually commuting they can be simultaneously localized with $\tilde{\phi}_{1}=\pi m_{1}$ and $\tilde{\theta}_{i}=\pi n_{i}$ with $m_{1}$ odd and $n_{1}$ even, or vice versa. Since the fields with tildes are $\mathbb{Z}_{4} \times \mathbb{Z}_{4}$ invariant we conclude that the system can be fully gapped without breaking symmetry. It is clear that this structure can be generalized to $\mathbb{Z}_{2 K} \times$ $\mathbb{Z}_{2 K}$ with $4 K$ flavors, one finds the same conclusion that the system can be gapped without breaking symmetry.

\section{DISCUSSION}

In conclusion we have proposed and developed a theoretical framework that allows us to determine if a given (edge) CFT can be gapped out or not without breaking a given set of symmetries. It is based on the modular invariance/non-invariance of the CFT with symmetry 
projection; it makes use of a way any 2D CFT couples to the background geometry (complex structure of the torus) and hence can be applied to a wide range of systems.

There are a number of merits to our approach; It does not rely on the presence/absence of a conserved U(1) charge such as particle number. Unlike topological invariants built from single-particle electron wave functions, our method does not rely on single-particle physics and hence is applicable to strongly interacting systems. It is simpler and more convenient than actually looking for all possible perturbations that can potentially gap out the edge theory on a case -by- case basis. For 2D SPT phases that have non-abelian quasiparticles there is no $K$-matrix formulation but our approach can be extended to such situations. For example, one could consider orbifolds of Wess-Zumino-Witten(WZW) models with discrete torsion.

We have demonstrated that our scheme indeed works for bosonic and fermionic SPT phases with $\mathbb{Z}_{K} \times \mathbb{Z}_{K}$ or $\mathbb{Z}_{K}$ symmetry. In particular, we have checked explicitly that for the cases when the modular invariance is achieved, one can find an interaction potential that can gap out the edge theory without breaking symmetry.

The validity of our approach based on the modular invariance is further supported by a complementary point of view proposed in Refs. 33 and 36 . In various cases, our method based on the modular invariance and the arguments in Refs. 33 and 36 that makes use of the fractional statistics in the bulk also lead to the same conditions for the "gappability" of the edge theory.

One immediate generalization of our work is to apply our method to symmetry-enriched topological phases, i.e., topologically ordered phases that have a set of symmetries. For example, our calculations for Bosonic SPT phases can be directly generalized to the case with $|\operatorname{det} K|>1$, which has ground state degeneracy. We have checked for a few simple cases with $|\operatorname{det} \mathrm{K}|>1$ that when the modular invariance is achieved we can construct an interaction potential to gap out the edge theory $\underline{51}$ Other interesting future work would be to consider SET phases with non-abelian symmetry and(or) non-Abelian statistics.

We close with a couple of comments. As discussed in the introduction, modular invariance is a global anomaly in CFT. On the other hand, it is interesting to note that in CFT a local anomaly associated with rescaling invariance occurs proportionately to the total central charge $c$. It is also instructive to note that in string theory conformal invariance is a constraint and $c$ is cancelled by working in a critical dimension. In condensed matter and statistical physics applications conformal invariance is a real symmetry (i.e., not a constraint) and the appearance of a local anomaly is a quantum effect which does not spoil the consistency of the theory (since there is no associated dynamical gauge degree of freedom).

- We have focussed entirely on modular (non)invariance on the torus. One may wonder if there are other constraints that come about at higher genus due to modular invariance and unitarity. Examples on torodial compactifications of string theory are explored in Ref. 52 , where it is shown that modular invariance and unitarity at genus 2 enforces more constraints on the phases with the various possible ways of achieving modular invariance corresponding to elements in the second group cohomology $H^{2}(G, U(1))$ for a finite Abelian group $G$. Perhaps one can make a connection between modular non-invariance and group cohomology as well, in particular the third cohomology which is relevant for the classification of 2D SPT phases. We will not pursue this issue further here.

\section{ACKNOWLEDGMENTS}

We would like to thank Michael Levin for useful discussion. SR thanks participants in Topological Phases of Matter Program at the Simons Center for Geometry and Physics for discussion. This work was supported in part by the National Science Foundation under grant No. DMR 1064319 at the University of Illinois (X.C.)

1 X.-G. Wen, Quantum Field Theory of Many-Body Systems, (Oxford University Press, Oxford, 2004).

2 The Quantum Hall Effect, edited by R. E. Prange and S. M. Girvin (Springer, New York, 1987).

3 Chetan Nayak, Steven H. Simon, Ady Stern, Michael Freedman, Sankar Das Sarma, Rev. Mod. Phys. 80, 1083 (2008).

4 M. Z. Hasan, and C. L. Kane, Rev. Mod. Phys. 82, 3045 (2010).

5 X.-L. Qi, and S.-C. Zhang, Rev. Mod. Phys. 83, 1057 (2011).

6 C. L. Kane and E. J. Mele, Phys. Rev. Lett. 95, 146802 (2005); Phys. Rev. Lett. 95, 226801 (2005).

7 B. A. Bernevig and S.-C. Zhang, Phys. Rev. Lett. 96, 106802 (2006).

8 B. A. Bernevig, T. Hughes and S.-C. Zhang, Science 314, 1757 (2006).

9 J. E. Moore and L. Balents, Phys. Rev. B 75, 121306(R) (2007).

10 R. Roy, Phys. Rev. B 79, 195322 (2009).

11 L. Fu, C. L. Kane, and E. J. Mele, Phys. Rev. Lett. 98, 106803 (2007).

12 L. Fu and C. L. Kane, Phys. Rev. B 76, 045302 (2007).

13 X.-L. Qi, T. L. Hughes and S.-C. Zhang, Phys. Rev. B 78, 195424 (2008).

14 A. P. Schnyder, S. Ryu, A. Furusaki, and A. W. W. Ludwig, Phys. Rev. B 78, 195125 (2008).

15 S. Ryu, A. Schnyder, A. Furusaki and A. W. W. Ludwig, New J. Phys. 12, 065010 (2010). 
16 A. Yu Kitaev, in Advances in Theoretical Physics: Landau Memorial Conference, Chernogolovka, Russia, 2008, edited by V. Lebedev and M. Feigel'man, AIP Conf. Proc. No. 1134 (AIP, Melville, NY, 2009), p. 22.

17 F. Pollmann, A. M. Turner, E. Berg, and M. Oshikawa, Phys. Rev. B 81, 064439 (2010), arXiv:0910.1811.

18 X. Chen, Z.-C. Gu, and X.-G. Wen, Phys. Rev. B 83, 035107 (2011)

19 N. Schuch, D. Perez-Garcia, and I. Cirac, Phys. Rev. B 84, 165139, (2011), arXiv:1010.3732

20 X. Chen, Z.-C. Gu, and X.-G. Wen, Phys. Rev. B 84, 235128 (2011).

${ }^{21}$ L. Fidkowski and A. Kitaev, Phys. Rev. B 81, 134509 (2010).

${ }^{22}$ L. Fidkowski and A. Kitaev, Phys. Rev. B 83, 075103 (2011).

23 A. M. Turner, F. Pollmann, and E. Berg, Phys. Rev. B 83, 075102 (2011).

24 Zheng-Cheng Gu, and Xiao-Gang Wen, Phys. Rev. B 80, 155131 (2009).

${ }^{25}$ Xie Chen, Zheng-Cheng Gu, Zheng-Xin Lin, and XiaoGang Wen, Phys. Rev. B 87, 155114 (2013).

26 Zheng-Cheng Gu, and Xiao-Gang Wen, arXiv:1201.2648.

27 M. Levin and A. Stern, Phys. Rev. Lett 103, 196803 (2009).

28 Titus Neupert, Luiz Santos, Shinsei Ryu, Claudio Chamon, and Christopher Mudry, Phys. Rev. B 84, 165107 (2011).

29 Yuan-Ming Lu, Ashvin Vishwanath Phys. Rev. B 86, 125119 (2012).

30 Yuan-Ming Lu and Ashvin Vishwanath, arXiv:1302.2634.

31 J.Wang, and X.-G. Wen, arXiv: 1212.4863

32 A. Kapustin and N. Saulina, Nucl. Phys. B 845, 393 (2011)

33 M. Levin, Phys. Rev. X 3, 021009 (2013).

34 Ling-Yan Hung and Yidun Wan, Phys. Rev. B 87, 195103(2013), arXiv: 1302.2951.

35 Meng Cheng and Zhen-Cheng Gu, arXiv:1302.4803.

36 M. Levin and Z. -C. Gu, Phys. Rev. B 86, 115109 (2012).

37 Z. -C. Gu and M. Levin, arXiv: 1304.4569.

38 Shinsei Ryu and Shou-Cheng Zhang, Phys. Rev. 85, 245132 (2012).
39 R. B. Laughlin, Phys. Rev. B 23, 5632 (1981).

40 L. Dixon, D. Friedan, E. Martinec, S. Shenker, Nucl. Phys. B, 282, 13 (1987).

41 L. Dixon, J. Harvey, C. Vafa, E. Witten, Nucl. Phys. B, 261, 678(1985).

42 An orbifold projection usually refers to a symmetry projection which leads to a space-time singularity in the string theory context. In this paper, we use the word orbifold for symmetry projections in general, which do not necessary generate a space-time singularity. More specfically, the type of projections we discuss in this paper include "shifted orbifolds" that do not give rise to a singularity (but are still called orbifolds).

43 K.S. Narain, M.H. Sarmadi and C. Vafa, Nucl. Phys. B 288, 551(1987); 356, 163 (1991).

44 Xiao-Liang Qi, New J. Phys. 15, 065002 (2013), arXiv:1202.3983.

45 Hong Yao and Shinsei Ryu, arXiv:1202.5805.

46 A. Cappelli, G. R. Zemba, Nucl. Phys. B 490, 595 (1997); A. Cappelli, L. S. Georgiev, G. R. Zemba, J. Phys. A 42 222001 (2009);

A. Cappelli, G. Viola, G. R. Zemba Ann. Phys. (NY) 325, 465 (2010);

Andrea Cappelli, Giovanni Viola, J. Phys. A 44, 075401 (2011)

47 Xiao-Liang Qi and Shou-Cheng Zhang, Phys. Rev. Lett. 101, 086802 (2008).

48 Orbifolding by a symmetry group is also known as gauging since from the point of view of target space physics we are using labels in a covering space to label states in the orbifolded theory. Therefore there is a redundancy in the labelling and hence a gauge symmetry.

49 J. L. Cardy, Nucl. Phys. B, 270, 186 (1986).

50 J. Polchinski, String Theory, (Cambridge University Press, Cambridge, 1998).

51 X.Chen, O.M. Sule, and S.Ryu (unpublished).

52 C. Vafa, Nucl. Phys. B 273, 592 (1986). 